\title{
Comparison of 2 electronic cowside tests to detect subclinical ketosis in dairy cows and the influence of the temperature and type of blood sample on the test results
}

\author{
M. Iwersen, ${ }^{* 1}$ D. Klein-Jöbstl, ${ }^{*}$ M. Pichler, ${ }^{*}$ L. Roland, ${ }^{*}$ B. Fidlschuster, ${ }^{*}$ I. Schwendenwein,† and M. Drillich* \\ ${ }^{*}$ Clinical Unit for Herd Health Management, Clinic for Ruminants, Department for Farm Animals and Veterinary Public Health, \\ University of Veterinary Medicine Vienna, 1210 Vienna, Austria \\ †Central Clinical Pathology Unit, Department for Pathobiology, University of Veterinary Medicine Vienna, 1210 Vienna, Austria
}

\begin{abstract}
The objective of this study was to determine the suitability of 2 electronic hand-held devices [FreeStyle Precision (FSP), Abbott GmbH \& Co. KG, Wiesbaden, Germany and GlucoMen LX Plus (GLX), A. Menarini GmbH, Vienna, Austria] for measuring $\beta$-hydroxybutyrate (BHBA) in dairy cows. Three experiments were conducted to evaluate (1) the diagnostic performance of the devices, (2) the effect of the type of blood sample, and (3) the influence of the ambient temperature on the determined results. A total of 415 blood samples from lactating Holstein and Simmental cows were collected and analyzed with both devices (whole blood) and in a laboratory (serum). Correlation coefficients between whole-blood and serum BHBA concentrations were highly significant, with $94 \%$ for the FSP and $80 \%$ for the GLX device. Based on thresholds for subclinical ketosis of 1.2 and $1.4 \mathrm{mmol}$ of BHBA/L, results obtained with the hand-held devices were evaluated by receiver operating characteristics analyses. This resulted in adjusted thresholds of 1.2 and 1.4 $\mathrm{mmol} / \mathrm{L}$ for the FSP and 1.1 and $1.3 \mathrm{mmol} / \mathrm{L}$ for the GLX device. Applying these thresholds, sensitivities were 98 and $100 \%$ for the FSP and 80 and $86 \%$ for the GLX device, respectively. Corresponding specificities were 90 and $97 \%$ for the FSP and 87 and $96 \%$ for the GLX device, respectively. Additionally, concentrations of BHBA were tested with both devices in whole blood, EDTA-added whole blood, and in their resulting serum and plasma, collected from 65 animals. Determined BHBA concentrations were similar within each device for whole and EDTA-added blood, and in serum and plasma, but differed between whole blood and serum and between EDTA-added blood and plasma. Blood samples with low $(0.4 \mathrm{mmol} / \mathrm{L})$, medium $(1.1 \mathrm{mmol} / \mathrm{L})$, and high $(1.6 \mathrm{mmol} / \mathrm{L})$ BHBA concentrations were
\end{abstract}

Received June 11, 2013.

Accepted September 2, 2013.

${ }^{1}$ Corresponding author: Michael.Iwersen@vetmeduni.ac.at stored between +5 to $+32^{\circ} \mathrm{C}$ and analyzed repeatedly at temperature levels differing by $4^{\circ} \mathrm{C}$. Additionally, devices and test strips were stored at equal conditions and used for measurement procedures. Storage temperature of the devices and test strips did not influence the differences between the results of the laboratory and the devices, whereas the temperature of the blood samples caused significant differences. Although the level of agreement between the laboratory and the GLX device was lower than for the laboratory and the FSP device, both devices are useful tools for monitoring subclinical ketosis in dairy cows. Due to their effects on the determined results, the type and temperature of the tested sample should be considered.

Key words: cow, ketosis, $\beta$-hydroxybutyrate, diagnostic test

\section{INTRODUCTION}

After parturition, the majority of all cows undergo a distinct period of negative energy balance. The reduction in feed intake shortly before parturition (Hayirli et al., 2002; Grummer et al., 2004) as well as the lagged increase in DMI within the first weeks of lactation causes a discrepancy between the energy required and consumed for lactation (Grummer, 2008). Some degree of negative energy balance and hence body fat mobilization with increased levels of NEFA is expected in the transition period (Ospina et al., 2010) and is a normal homeorhetic adaption of the cow with the onset of lactation (Bauman and Currie, 1980; Bell, 1995; Duffield et al., 2009). Excessive body fat mobilization, exceeding the liver's capacity to completely oxidize NEFA, is associated with high concentrations of ketones, as BHBA, acetone, and acetoacetate (AcAc). These excessive levels are indicative of maladaptation of the energy metabolism during early lactation (Suriyasathaporn et al., 1999; Duffield et al., 2009; LeBlanc, 2010).

Elevated concentrations of BHBA in blood are associated with an increased risk for periparturient metabolic disorders or infectious diseases (Cook et al., 2006a; 
Dubuc et al., 2010; Suthar et al., 2013). Furthermore, negative associations of elevated BHBA concentrations on milk production (Duffield et al., 2009; Chapinal et al., 2012) and reproductive performance (Walsh et al., 2007a; Chapinal et al., 2012) have been reported. Subsequently, affected cows are at higher risk to be removed from the herd within early lactation (Oetzel, 2004).

In the periparturient period, up to $50 \%$ of the animals suffer from metabolic or infectious diseases and approximately $75 \%$ of diseases in dairy cows occur within this time frame (LeBlanc, 2010). Therefore, monitoring high-producing dairy herds for subclinical diseases in the periparturient period (e.g., for ketosis and hypocalcaemia) is considered to be beneficial and is recommended by several authors (Geishauser et al., 1998; Oetzel, 2004; Cook et al., 2006a,b; LeBlanc, 2010).

Subclinical ketosis (SCK) within the first 2 wk of lactation has been defined as serum BHBA concentrations exceeding 1.2 and $1.4 \mathrm{mmol} / \mathrm{L}$ in serum (Duffield et al., 1998; Cook et al., 2006a; Duffield et al., 2009) without any clinical signs of the disease. The gold standard test for detecting SCK is to determine the concentration of BHBA in serum or plasma (Duffield et al., 1998), because of its stable characteristics in blood, compared with acetone and AcAc (Herdt, 2000). Various methods have been described for cowside BHBA testing in urine, milk and blood, with different test characteristics (Geishauser et al., 2000; Carrier et al., 2004; Iwersen et al., 2009). Recent studies based on blood BHBA concentrations reported incidences of SCK from d 3 to 16 of lactation between $26.4 \%$ and $55.7 \%$ in 4 North American dairy farms (McArt et al., 2012). Suthar et al. (2013) presented an overall prevalence of SCK of $21.8 \%$ ( 11.2 to $36.6 \%$ ) for 10 European countries within 2 to $15 \mathrm{~d}$ after parturition using a threshold of $\geq 1.2 \mathrm{mmol}$ of $\mathrm{BHBA} / \mathrm{L}$ in blood.

Within the last few years, excellent test characteristics were published for electronic hand-held devices, which were initially developed for the human medicine market. To our knowledge, so far, only devices distributed by Abbott Diabetes Care (Witney, UK) with differing labels were evaluated for BHBA testing in dairy cows (Jeppesen et al., 2006; Heuwieser et al., 2007; Iwersen et al., 2009; Voyvoda and Erdogan, 2010) and sheep (Panousis et al., 2012; Pichler et al., 2012).

The present study covers 3 objectives: (1) 2 new commercially available electronic hand-held devices [FreeStyle Precision (FSP), Abbott GmbH \& Co. KG, Wiesbaden, Germany and GlucoMen LX Plus (GLX), A. Menarini GmbH, Vienna, Austria) were evaluated for detection of subclinical ketosis in dairy cows, (2) the effect of the type of blood sample (whole blood,
EDTA-anticoagulated blood, serum, and plasma) was determined, and (3) the effects of the storage temperature of the device, the test strips, and the blood sample on the results of the devices were evaluated.

\section{MATERIALS AND METHODS}

The study was approved by the institutional ethics committee and the national authority according to $\S 8 f f$ of the Law for Animal Experiments [Tierversuchsgesetz (TVG); BMWF-68.205/0132-II/3b/2011]. Three experiments were conducted between January and December 2012. For all experiments, the 2 electronic hand-held devices (FSP and GLX) for determination of BHBA in whole blood were used. Each test system consists of an electronic hand-held meter and electrochemical test strips [FreeStyle Precision $\beta$-Ketone (Abbott Diabetes Care, Witney, UK) and GlucoMen LX $\beta$-Ketone Sensor (A. Menarini $\mathrm{GmbH}$ )]. In contrast to the alreadyevaluated Precision Xtra device (Abbott GmbH \& Co. $\mathrm{KG})$, calibration procedures of the meters before starting measurements are not necessary anymore. After inserting the test strips into the meter, a small amount of whole blood $(1.5 \mu \mathrm{L}$ for the FSP and $0.8 \mu \mathrm{L}$ for the GLX device) is applied to the front edge of the sample application zone of the sensor, starting a chemical reaction within the test strips as follows: the BHBA in the blood sample is oxidized to AcAc in presence of the enzyme BHBA dehydrogenase, with the concomitant reduction of $\mathrm{NAD}^{+}$to NADH. The NADH is reoxidized to $\mathrm{NAD}^{+}$by a redox mediator. This chemical reaction releases electrons, generating a small current, which is directly proportional to the BHBA concentration in the sample. For both devices, test results are presented 10 $\mathrm{s}$ after application of the blood, presented as a digital value on the display of the meters. Referring to the manufacturer's manuals, the minimum operating temperature for both devices is $+4^{\circ} \mathrm{C}$.

\section{Experiment 1}

The Teaching and Research Farm (farm 1) of the University of Veterinary Medicine, Vienna (Austria), with approximately 80 Simmental cows, and a commercial dairy farm (farm 2) in Mecklenburg-Vorpommern, Germany, with approximately 600 Holstein-Friesian cows were used as study sites. Animals were housed in freestall barns with rubber mats on concrete floors in farm 1, and on slotted concrete floors in farm 2. Both farms were equipped with deep-bedded cubicles, filled either with straw (farm 1) or sawdust (farm 2). In both farms, a TMR was fed that was offered 8 times per day by an automatic feeding system (Trioliet-Mullos BV, Oldenzaal, the Netherlands) in farm 1, and twice per 
day by a mixer wagon (Sgariboldi Deutschland, Gültstein, Germany) in farm 2. For both farms, rations were balanced by a dairy nutritionist to meet the energy and nutrient requirements for dairy cows as recommended by the German Society of Nutrition Physiology (GfE, 2001) as well as possible. Herd average ECM production (based on $4.0 \%$ butterfat and $3.4 \%$ protein) was $8,082 \mathrm{~kg}(2 \times$ milking) for farm 1 and $10,808 \mathrm{~kg}(3 \times$ milking) for farm 2.

A total of 425 blood samples were taken from cows within the first 3 mo of lactation in farm $1(\mathrm{n}=63)$ and at routine herd health checks between the first and sixth week of lactation in farm $2(\mathrm{n}=362)$. This time frame was chosen to ensure obtaining a variety of BHBA concentrations. Blood samples were drawn from a coccygeal vessel using 9-mL vacuum tubes with clot enhancer (Vacuette; Greiner Bio-One GmbH, Kremsmünster, Austria) with a 20-G needle (Hypodermic Luer Needle, 20G × 1 1/2"; Greiner Bio-One GmbH). Within $60 \mathrm{~s}$ after collection, the whole blood was tested in random order with both devices by dipping the sensor of the test strips directly onto the surface of the blood in the tube. Preassigned data capture forms were used to record the values displayed on the meters.

After clotting for approximately $2 \mathrm{~h}$ at room temperature, blood samples were centrifuged at 2,200 $\times g$ for 10 min (Eppendorf Centrifuge 5804; Eppendorf AG, Hamburg, Germany). Supernatant serum was stored in 2 aliquots of $2 \mathrm{~mL}$ each in microtubes (Mikro-Schraubröhrchen $2 \mathrm{~mL}$; Carl Roth GmbH \& Co. KG, Karlsruhe, Germany) at $-25^{\circ} \mathrm{C}$ until analysis at the Central Diagnostic Unit (CDU) of the University of Veterinary Medicine. The concentration of BHBA in serum was measured in the laboratory using a colorimetric enzymatic reaction (Ranbut D-3-hydroxybutyrate; Randox Laboratories Ltd., Antrim, UK) with an automated wet chemistry analyzer (Cobas 6000/501c; Roche Diagnostics International AG, Rotkreuz, Switzerland), and was used as the gold standard.

Strictly spoken, reference limits are only valid for single laboratories under their specific conditions (e.g., equipment, reagents, and staff). Therefore, to validate the laboratory results of the CDU, a subset of 212 samples, covering the complete range of BHBA concentrations measured with the FSP device of $0.2 \mathrm{mmol} / \mathrm{L}$ up to $3.2 \mathrm{mmol} / \mathrm{L}$, were shipped to a second commercial laboratory (Synlab.vet, Berlin, Germany) that analyzed samples for BHBA in prior studies (Iwersen et al., 2009). Additionally, 13 aliquot samples of 1 animal were shipped to both laboratories to evaluate their particular intraassay variability.

Based on determination with the FSP device, 3 samples of low $(0.4 \mathrm{mmol} / \mathrm{L})$, medium $(1.1 \mathrm{mmol} / \mathrm{L})$, and high $(1.6 \mathrm{mmol} / \mathrm{L})$ concentration of BHBA were used to evaluate the intra- and interassay coefficients of variation for the 2 electronic devices. For this purpose, EDTA-added blood samples were measured 10 times each with 1 device (intraassay) or with 10 different devices (interassay).

\section{Experiment 2}

The effect of the type of blood sample on the results obtained with the 2 hand-held meters was tested. For this, 65 animals already used in experiment 1 on farm 1 were used for collection of whole- and EDTA-blood samples, leaving the needle in the same position. Immediately after collection, samples were analyzed using the 2 hand-held meters. After clotting of whole blood, both samples were centrifuged (for details, see experiment 1) and supernatant serum and plasma, respectively, was harvested and analyzed for BHBA concentration with both hand-held devices. Serum samples were sent for laboratory analysis to use as the gold standard in this experiment.

\section{Experiment 3}

The effects of the temperature of the devices, the test strips, and the blood samples were analyzed. As described for experiment 1, 3 blood samples with low, medium, and high concentrations of BHBA, measured at room temperature $\left(22^{\circ} \mathrm{C}\right)$ with the FSP device in whole blood, were used. Blood-sampling procedures were identical as described for experiment 1, but coated EDTA tubes (Vacuette EDTA; Greiner Bio-One $\mathrm{GmbH}$ ) were used to prevent clotting. Each sample was split into 9 aliquots of $2 \mathrm{~mL}$ each into microtubes to prevent any bias resulting from volatizing BHBA by repeated opening of the sample tubes.

Samples were either stored at room temperature and at chilled or heated storage conditions. Additionally, the test devices, as well as the test strips were stored at identical conditions as described before. To simulate different situations in veterinary practice regarding the storage of devices, test strips, and samples, the following 4 scenarios were created: (1) the blood samples were stored at systematically varied temperature conditions, whereas the devices and test strips were stored at room temperature; (2) the samples, devices, and test strips were kept at various temperatures; (3) the samples, devices, and test strips were kept at room temperature; and (4) samples were kept at room temperature, whereas the devices and test strips were stored at various temperature conditions.

Starting with room temperature, samples and (or) test devices and test strips were cooled stepwise by decrements of $4^{\circ} \mathrm{C}$ down to $+4^{\circ} \mathrm{C}$ using a mobile refrigerator 
box (CoolFreeze CF-50; Dometic Waeco International GmbH, Emsdetten, Germany). Furthermore, samples or devices, or both, were kept under heated conditions stepwise by $4^{\circ} \mathrm{C}$, up to $+32^{\circ} \mathrm{C}$ in a water bath, stored in waterproof plastic bags. To ensure that the samples reached the designated temperature, the tip of the cable probe of a digital laboratory thermometer (LT-102; TFA Dostmann GmbH \& Co. KG, WertheimReicholzheim, Germany) was stored in 1 reference sample. By reaching the designated temperature, 1 of the 9 aliquots, as described above, was measured with both devices and dumped afterward.

\section{Statistical Analyses}

Data were analyzed using SPSS Statistics 20.0 for Windows (IBM Deutschland GmbH, Ehningen, Germany) and MedCalc for Windows (version 12.4; MedCalc Software bvba, Ostend, Belgium). Serum concentrations of BHBA analyzed at the CDU of the University of Veterinary Medicine were defined as the gold standard. For samples analyzed at the CDU and in the commercial laboratory, correlation coefficients (Spearman) for BHBA in serum were calculated between both laboratories. Coefficients of variation were calculated as standard deviation divided by the mean times 100 . Intraassay coefficients of variation were calculated for both laboratories and for the 2 hand-held devices. Furthermore interassay coefficients of variation were calculated for the 2 devices. The level of significance for all tests was set at $P=0.05$.

In experiment 1 , for each hand-held device, Spearman correlation coefficients (r) were calculated between the BHBA concentration analyzed at the CDU and the value displayed on the meter for whole blood. Using only correlation coefficients for method comparison studies can be inappropriate. Hence, the agreement between each meter and the gold standard was analyzed graphically using the method described by Bland and Altman (1986).

To identify best thresholds to distinguish between healthy and animals suffering from SCK, receiver operating characteristics (ROC) analyses were performed for each device. As part of the ROC analyses, the truepositive rate [sensitivity (Se)] is plotted against the false-positive rate $[1$ - specificity $(\mathbf{S p})]$ for the complete range of cut-off points (Swets, 1988). The closer the ROC plot is to the upper left corner of the coordinate system, the greater the overall accuracy of the test. The area under the ROC curve (AUC) is used to rate the quality of the analyzed threshold. If the curve lies along the major diagonal line $(\mathrm{AUC}=0.5)$, the trueand false-positive rates are equal (Swets, 1988); thus, the used threshold is not able to distinguish between healthy and animals suffering from SCK. With perfect test discrimination $(\mathrm{AUC}=1.0)$, the curve follows the left and upper axes, such that the true-positive rate is 1.0 for all values of the false-positive rate (Swets, 1988).

Executing the ROC analyses, a serum BHBA concentration of $\geq 1.2 \mathrm{mmol} / \mathrm{L}$ measured by the wet chemistry standard method was used as the classification variable. Cows with BHBA concentrations $\geq 1.2 \mathrm{mmol} / \mathrm{L}$ were classified as suffering from SCK. Based on the optimized threshold for the 2 hand-held devices, means and $95 \%$ confidence intervals of the test characteristics Se and Sp were calculated. The Se was defined as the proportion of samples with serum BHBA concentrations $\geq 1.2 \mathrm{mmol} / \mathrm{L}$ correctly diagnosed as positive by the test. The Sp was calculated as the proportion of samples with concentrations less than $1.2 \mathrm{mmol} / \mathrm{L}$ correctly diagnosed as negative by the test. Additionally, ROC analyses were performed by using a BHBA concentration in serum of $\geq 1.4 \mathrm{mmol} / \mathrm{L}$ as classification variable for SCK.

In experiment 2, the Wilcoxon signed-rank test for related samples was chosen to test the agreement between the BHBA concentrations analyzed with the hand-held meters in whole blood, EDTA-added blood, serum, and plasma, respectively, and the laboratory serum concentration. The influence of the temperature on the results of the hand-held meters in experiment 3 was determined by analysis of covariances, using the difference between the laboratory result and the meter value as the dependent variable and the temperature of the sample and (or) devices and test strips as covariates.

\section{RESULTS}

The coefficient of correlation between the BHBA concentration determined at the CDU and the commercial laboratory was $99.2 \%(P<0.01)$. The mean difference in BHBA concentration between both laboratories was $0.01 \pm 0.05 \mathrm{mmol} / \mathrm{L}$. The intraassay coefficients of variation were similar in both laboratories, with $2.40 \%$ for the CDU and $2.37 \%$ for the commercial laboratory.

\section{Experiment 1}

All of the 425 blood samples collected in experiment 1 could be analyzed with the FSP device, whereas 25 $(5.9 \%)$ of the samples tested with the GLX device resulted in an E4-failure, indicating a "hematocrit level that exceeded the allowed operating range," referring to the manufacturer's manual. These 25 samples were tested for a second time with the GLX device, but in $10(2.4 \%)$ samples no result could be determined, leading to 415 analyzable sample pairs in total. Of these 415 samples analyzed at the CDU, 50 (12.0\%) 
exceeded a threshold of $\geq 1.2 \mathrm{mmol} / \mathrm{L}$ and $29(7.0 \%)$ of $\geq 1.4 \mathrm{mmol} / \mathrm{L}$ of BHBA in serum.

Coefficients of correlation for BHBA concentrations determined with the hand-held devices and at the CDU were $94.0 \%(P<0.01)$ for the FSP and $80.3 \%(P<$ $0.01)$ for the GLX device.

Intraassay and interassay coefficients of variation were below $10 \%$ for both devices, except for low BHBA concentrations tested with the GLX device (13.2 and $15.0 \%$; Table 1). On average, the intraassay coefficients of variation were $5.7 \%$ for the FSP and $7.0 \%$ for the GLX device. The average interassay coefficients of variation were also similar for both devices, with $5.8 \%$ for the FSP and $4.9 \%$ for the GLX device. Stated by the laboratory, the mean intraassay coefficients of variation, determined at routine quality assurance testing of the wet chemistry methods, were $3.82 \%$ at a BHBA concentration of $0.27 \mathrm{mmol} / \mathrm{L}$ and $1.95 \%$ at a concentration of $1.2 \mathrm{mmol} / \mathrm{L}$.

A summary of parameters related to the level of agreement between the results of the hand-held meters and the CDU are presented in Table 2. Whereas the FSP device overestimated the serum BHBA concentration $(+0.04 \pm 0.15 \mathrm{mmol} / \mathrm{L} ; P<0.01)$, the GLX device underestimated the laboratory results $(-0.12 \pm$ $0.22 \mathrm{mmol} / \mathrm{L} ; P<0.01$ ). Overall variance was 2 -fold greater for the GLX device $(26.6 \%)$ compared with the FSP device (13.3\%). The percentage of samples with a variance $\geq 20 \%$ was approximately $59 \%$ for the GLX and $23 \%$ for the FSP device. Plotting the differences in the BHBA concentrations measured with each handheld device and the gold standard against their mean revealed greater agreement for the FSP compared with the GLX device (Figure 1; Table 2).

Receiver operating characteristics analyses (Figure 2) using BHBA concentrations of 1.2 and $1.4 \mathrm{mmol} / \mathrm{L}$, determined at the $\mathrm{CDU}$ as thresholds to distinguish between healthy and animals suffering from SCK, were performed. The resulting corresponding thresholds for the hand-held devices are presented in Table 3. Assuming a concentration of BHBA in serum of 1.2 or $1.4 \mathrm{mmol} / \mathrm{L}$ as indicative for SCK, thresholds should be adapted for the GLX device by decreasing it by $0.1 \mathrm{mmol} / \mathrm{L}$.

As shown by the AUC (Figure 2), both hand-held meters were eligible to distinguish between healthy cows and animals suffering from SCK. Applying a BHBA concentration in serum of $1.2 \mathrm{mmol} / \mathrm{L}$ as the threshold for SCK, the AUC were $99 \%$ for the FSP and $94 \%$ for the GLX device. The difference of 5 percentage points was significant $(P<0.01)$ within the pairwise comparison of the 2 AUC (Figure 2, top). With a threshold of $1.4 \mathrm{mmol} / \mathrm{L}$ of BHBA in serum, the AUC presented on the bottom of Figure 2 with $98 \%$ for the GLX and $100 \%$ for the FSP device did not differ $(P=0.06)$.

Sensitivities and $\mathrm{Sp}$ for both devices applying the thresholds estimated by ROC analyses are presented in Table 3. For thresholds of 1.2 and $1.4 \mathrm{mmol} / \mathrm{L}$ of BHBA, both Se and Sp were numerically greater for the FSP compared with the GLX device.

\section{Experiment 2}

The differences between the gold standard and the BHBA concentrations measured with both devices in whole blood, EDTA-anticoagulated blood, serum, and plasma are presented as boxplots in Figure 3. The BHBA concentrations analyzed with the FSP in whole blood and EDTA-anticoagulated whole blood resulted in slightly greater $(P<0.01)$ BHBA concentrations compared with the laboratory results in serum. Concentrations of BHBA analyzed with the FSP in serum and plasma led to greater concentrations compared with the laboratory serum results $(P<0.01$; Table 4$)$.

Table 1. Intraassay and Interassay CV for FreeStyle Precision (Abbott GmbH \& Co. KG, Wiesbaden, Germany) and GlucoMen LX Plus (A. Menarini GmbH, Vienna, Austria) devices within low, medium, and high BHBA concentrations

\begin{tabular}{|c|c|c|c|c|c|c|c|c|}
\hline \multirow{2}{*}{ Parameter } & \multicolumn{8}{|c|}{ BHBA concentration $^{1}$} \\
\hline & \multicolumn{4}{|c|}{ FreeStyle Precision device } & \multicolumn{4}{|c|}{ GlucoMen LX Plus device } \\
\hline \multicolumn{9}{|l|}{ Intraassay } \\
\hline Mean (mmol/L) & 0.58 & 1.46 & 2.02 & 1.35 & 0.32 & 1.08 & 1.28 & 0.89 \\
\hline $\mathrm{SD}(\mathrm{mmol} / \mathrm{L})$ & 0.04 & 0.08 & 0.10 & 0.08 & 0.04 & 0.04 & 0.10 & 0.06 \\
\hline Mean (mmol/L) & 0.52 & 1.44 & 2.02 & 1.33 & 0.28 & 1.14 & 1.38 & 0.93 \\
\hline $\mathrm{SD}(\mathrm{mmol} / \mathrm{L})$ & 0.04 & 0.08 & 0.10 & 0.08 & 0.04 & 0.05 & 0.04 & 0.05 \\
\hline $\mathrm{CV}(\%)$ & 8.1 & 5.9 & 5.1 & 5.8 & 15.0 & 4.5 & 3.1 & 4.9 \\
\hline
\end{tabular}

${ }^{1}$ Based on measurement with FreeStyle Precision (low: $0.4 \mathrm{mmol} / \mathrm{L}$; medium: $1.1 \mathrm{mmol} / \mathrm{L}$; high: $1.6 \mathrm{mmol} / \mathrm{L}$ ).

${ }^{2}$ Coefficient of variation $=\mathrm{SD} \times 100 /$ mean; deviations are caused by rounding. 
Table 2. Differences between GlucoMen LX Plus (A. Menarini GmbH, Vienna, Austria) and FreeStyle Precision (Abbott GmbH \& Co. KG, Wiesbaden, Germany) devices compared with serum BHBA concentrations measured in experiment 1

\begin{tabular}{|c|c|c|}
\hline \multirow[b]{2}{*}{ Parameter } & \multicolumn{2}{|c|}{ Device } \\
\hline & $\begin{array}{c}\text { GlucoMen } \\
\text { LX Plus }\end{array}$ & $\begin{array}{l}\text { FreeStyle } \\
\text { Precision }\end{array}$ \\
\hline Number of samples & 415 & 415 \\
\hline \multicolumn{3}{|l|}{ Mean $\pm \mathrm{SD}(\mathrm{mmol} / \mathrm{L})$} \\
\hline Laboratory & $0.87 \pm 0.45$ & $0.87 \pm 0.45$ \\
\hline Hand-held device & $0.75 \pm 0.44$ & $0.91 \pm 0.53$ \\
\hline Difference mean $\pm \mathrm{SD}(\mathrm{mmol} / \mathrm{L})$ & $-0.12 \pm 0.22$ & $+0.04 \pm 0.15$ \\
\hline Variance $^{1}(\%)$ & 26.6 & 13.3 \\
\hline \multicolumn{3}{|l|}{ Percentage of pairs with } \\
\hline Variance $<10 \%$ & 19.8 & 47.0 \\
\hline Variance $<20 \%$ & 40.7 & 76.6 \\
\hline Variance $\geq 20 \%$ & 59.3 & 23.4 \\
\hline
\end{tabular}

${ }^{1}$ Based on mean of serum BHBA concentration analyzed at the laboratory of the University of Veterinary Medicine, Vienna (Austria) and the concentration measured with GlucoMen LX Plus or FreeStyle Precision devices.

The measurements of the BHBA concentration with the GLX device in whole and EDTA-added blood underestimated $(P<0.01)$ the laboratory results by a median of $0.3 \mathrm{mmol} / \mathrm{L}$ (Table 4; Figure 3). Using the GLX device, BHBA concentrations in serum were slightly overestimated $(P<0.01)$, whereas concentrations in plasma were consistent $(P=0.06)$ with the laboratory results.

Nonparametric testing with the Wilcoxon signed-rank test showed no differences between the BHBA concentration determined with the hand-held meters in whole blood or EDTA-added blood ( $P=0.06$ for FSP; $P=$ 0.99 for GLX). The Spearman correlation coefficients of $94 \%$ for the FSP and $87 \%$ for the GLX device between the paired samples were significant $(P<0.01$ for both devices). Because of these results, EDTA samples were chosen for experiment 3 to prevent clotting, allowing testing for a longer period.

\section{Experiment 3}

In experiment 3, EDTA-added blood samples were used to evaluate the effect of differing ambient temperatures on the results of the meters. Instead of presenting invalid BHBA concentrations by cooling the devices and test strips down to $+4^{\circ} \mathrm{C}$, error messages were displayed for both devices, indicating that the temperature was out of range. Therefore, the results at a minimum temperature of $+5^{\circ} \mathrm{C}$ were used for statistical analyses.

The results of the analysis of covariances are presented in Table 5. For both devices, the storage temperature of the device and the test strips had no significant influence (FSP: $P=0.99$; GLX: $P=0.55$ ) on the difference between the laboratory results and the results determined with each particular device. The temperature of the blood sample had a significant effect $(P<0.01$ for both devices) on the difference between the BHBA concentration measured with the hand-held devices and the laboratory result, as shown in Figure 4. The regression lines for the FSP and GLX devices (Figure 4) run almost parallel [sample temperature: regression coefficient $(\mathrm{B})=-0.012$ for $\mathrm{FSP}, \mathrm{B}=-0.008$ for GLX; e.g., increasing the temperature by $1^{\circ} \mathrm{C}$ will decrease the difference between laboratory and FSP by $0.012 \mathrm{mmol} / \mathrm{L}$ and GLX by $0.008 \mathrm{mmol} / \mathrm{L}]$, but with differing $\mathrm{y}$-axis intercepts (intercept: $\mathrm{B}=0.42$ for FSP, $\mathrm{B}=0.07$ for GLX).

Increasing the temperature of the EDTA-anticoagulated samples within a range of +5 to $+32^{\circ} \mathrm{C}$ resulted in a decreasing difference in BHBA concentrations between the laboratory and the results analyzed with the FSP from approximately +40 to $0 \%$. Within the same temperature range, the difference between the laboratory and the GLX device increased from approximately +1 to $-20 \%$ (Figure 4 ). Mean absolute differences in BHBA measurements between +5 and $+32^{\circ} \mathrm{C}$ for low $(0.4 \mathrm{mmol} / \mathrm{L})$, medium $(1.1 \mathrm{mmol} / \mathrm{L})$, and high $(1.6 \mathrm{mmol} / \mathrm{L})$ BHBA concentrations were $0.2,0.2$, and $0.4 \mathrm{mmol} / \mathrm{L}$ for the FSP device and $0.1,0.5$, and 0.4 $\mathrm{mmol} / \mathrm{L}$ for the GLX device.

\section{DISCUSSION}

To our knowledge, this is the first study evaluating the hand-held devices FreeStyle Precision, a refined version of the previously evaluated Precision Xtra, and the device GlucoMen LX Plus for detection of SCK in dairy cows. Additionally, the influence of the type of blood sample and the effect of the temperature on 

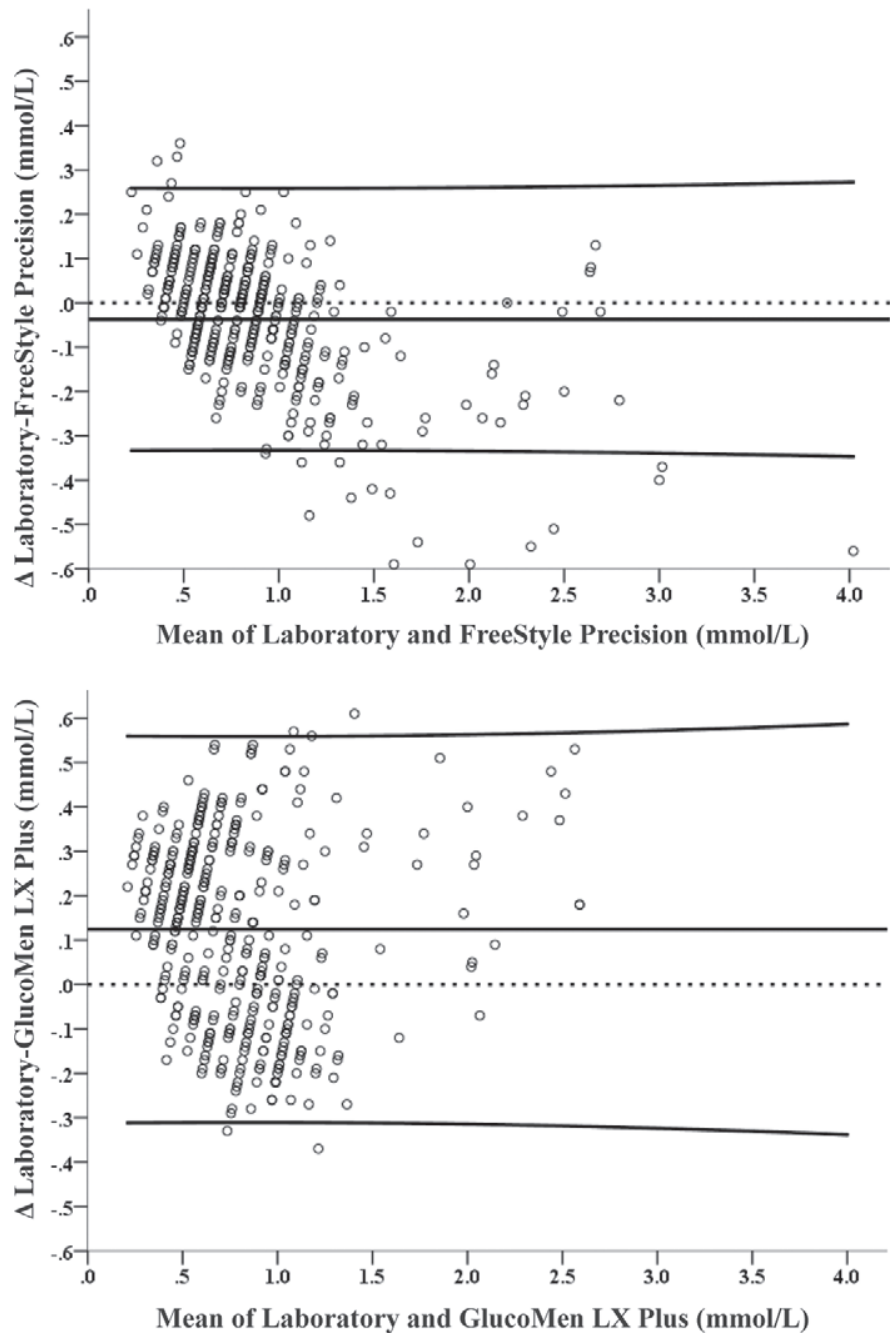

Figure 1. Differences in BHBA concentrations measured either with the GlucoMen LX Plus (A. Menarini GmbH, Vienna, Austria; bottom) or FreeStyle Precision (Abbott GmbH \& Co. KG, Wiesbaden, Germany; top) device and the gold standard against their mean. The solid line in the middle represents the mean; the upper and lower solid lines represent the mean $\pm 2 \mathrm{SD}$.

the results obtained with these hand-held meters were investigated.

Reference values are technically only valid for single laboratories because of their specific conditions of equipment, reagents, target population, and staff training (Quiroz-Rocha et al., 2009). To evaluate the range of this possible factor, a subset of serum samples were tested at the CDU and a commercial laboratory. The BHBA concentrations measured at both sites were highly correlated at $99.2 \%$. Mean differences between the BHBA concentrations determined at both laboratories were low $(0.01 \pm 0.05 \mathrm{mmol} / \mathrm{L})$, indicating that the results of the CDU are comparable to the laboratory used in prior studies. The observed overall intraassay
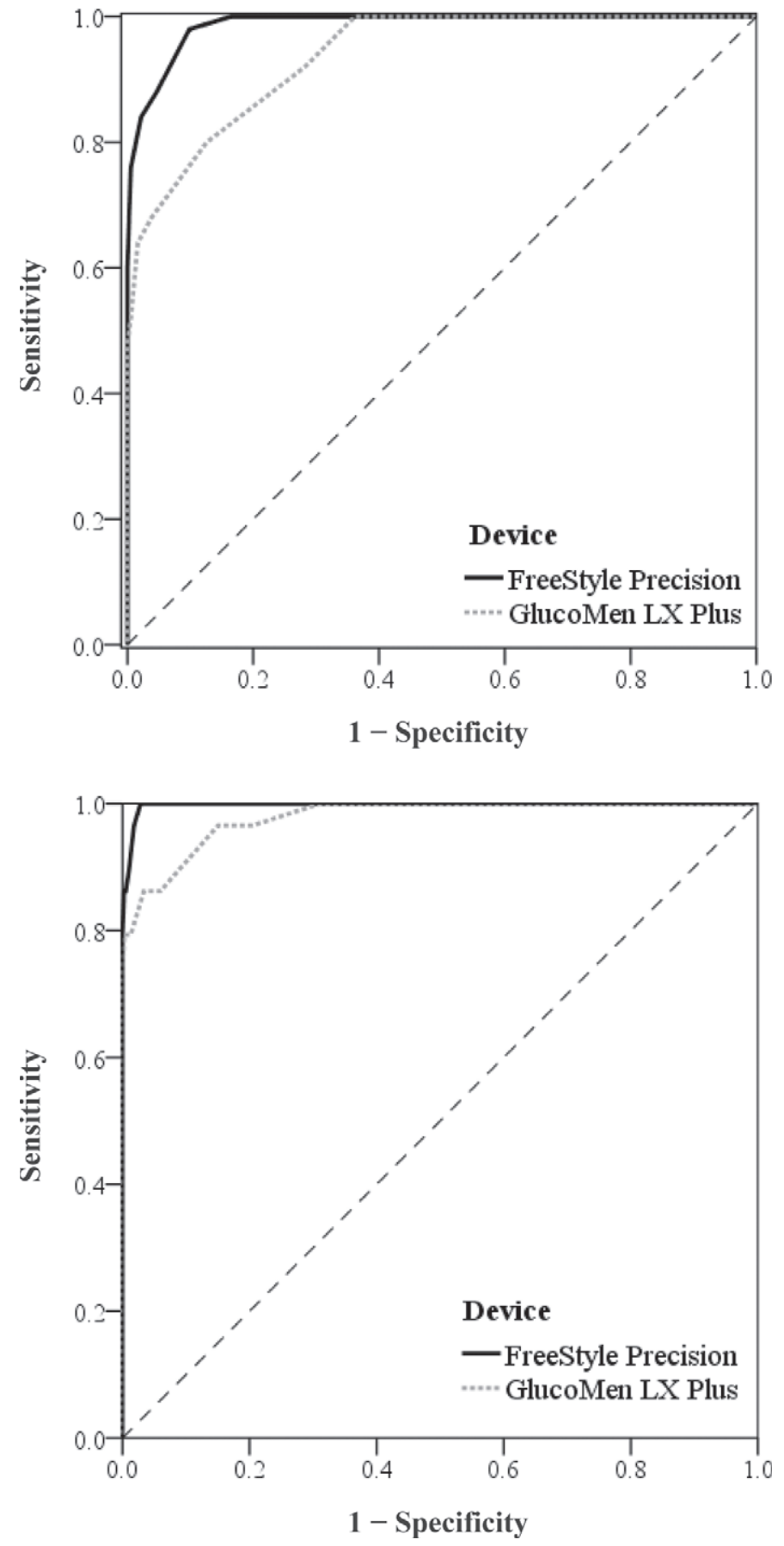

Figure 2. Receiver operating characteristics (ROC) analyses for the FreeStyle Precision (Abbott GmbH \& Co. KG, Wiesbaden, Germany) and GlucoMen LX Plus (A. Menarini GmbH, Vienna, Austria) devices, using serum BHBA concentrations of 1.2 (top) or $1.4 \mathrm{mmol} / \mathrm{L}$ (bottom) as thresholds for subclinical ketosis.

coefficients of variation $(5.7 \%$ for the FSP and $7.0 \%$ for the GLX device) were good for both hand-held meters, indicating good precision in terms of repeatability and reproducibility. 
Table 3. Corresponding thresholds and performance of 2 hand-held devices for detection of ketosis to serum BHBA concentrations of 1.2 or $1.4 \mathrm{mmol} / \mathrm{L}^{1}$

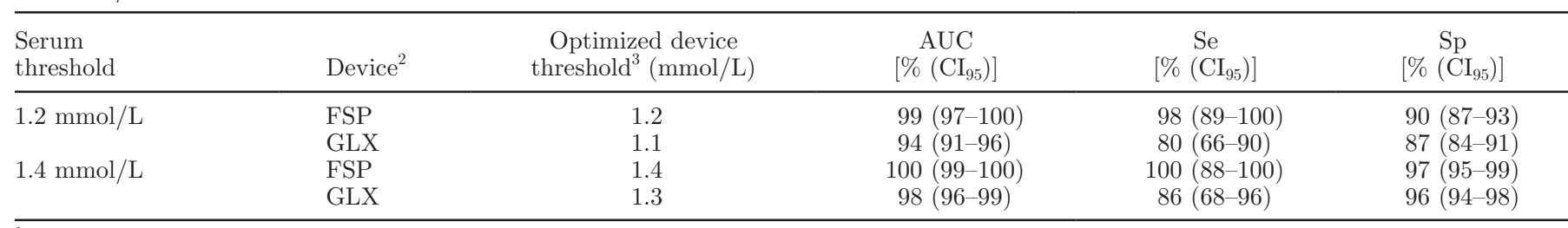

${ }^{1} \mathrm{AUC}=$ area under the receiver operating characteristics $(\mathrm{ROC})$ curve; Se $=$ sensitivity (proportion of ketotic cows that test positive); Sp $=$ specificity (proportion of nonketotic cows that test negative) $\mathrm{CI}_{95}=95 \% \mathrm{CI}$.

${ }^{2}$ FSP = FreeStyle Precision (Abbott GmbH \& Co. KG, Wiesbaden, Germany); GLX = GlucoMen LX Plus (A. Menarini GmbH, Vienna, Austria).

${ }^{3}$ Based on ROC analyses.

The intention of the present study was not to evaluate the prevalence of SCK for the 2 farms used in this study, but to evaluate the suitability of the 2 hand-held devices, covering a wide range of BHBA concentrations. Therefore, the sampling period was extended, compared with the cited prevalence studies, up to $100 \mathrm{~d}$ after parturition. Furthermore, animals could be tested several times within the given time frame, leading to incomparable parameters with the cited studies. Thus, the determined overall proportion of serum BHBA concentration $\geq 1.2$ and $\geq 1.4 \mathrm{mmol} / \mathrm{L}$ of 12.0 and $7.0 \%$, respectively, should not be overinterpreted. These proportions are considerably lower than those reported in previous studies on farms in the United States, Turkey,

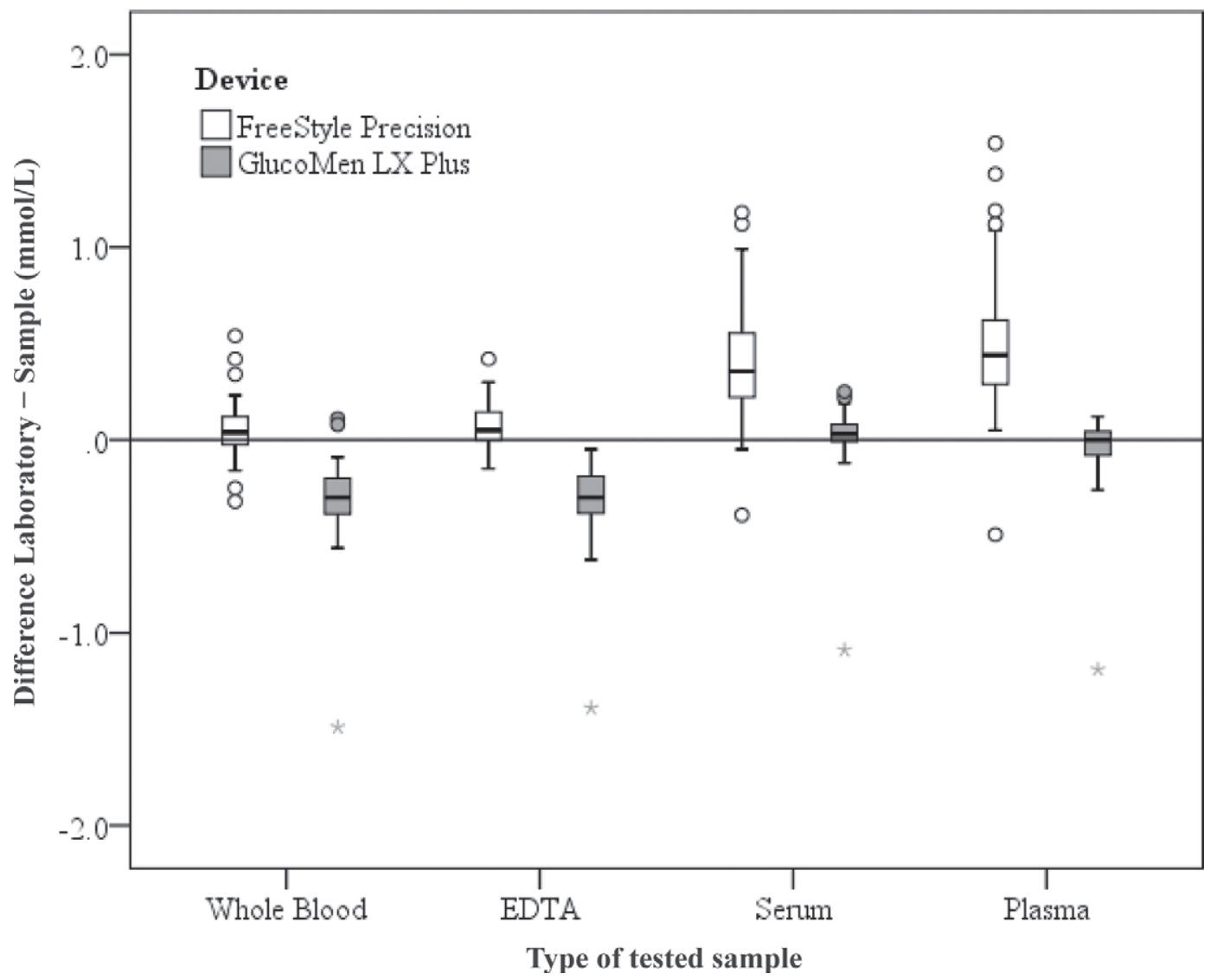

Figure 3. Differences between the BHBA concentrations in serum analyzed at the laboratory (gold standard) and the concentrations measured with the hand-held devices FreeStyle Precision (Abbott GmbH \& Co. KG, Wiesbaden, Germany) and GlucoMen LX Plus (A. Menarini GmbH, Vienna, Austria) in whole blood, EDTA-added blood, serum, and plasma. The heavy black line inside each box marks the median (50th percentile); lower and upper hinges mark the 25th and 75th percentiles. Whiskers end at the smallest and largest statistical values that are not outliers; outliers and extreme values are designated by $\bigcirc$ and *. 
Table 4. Correlation coefficients and median differences between the BHBA concentrations analyzed at the laboratory in whole blood, EDTA blood, serum, and plasma using 2 hand-held meters

\begin{tabular}{|c|c|c|c|c|c|c|c|c|}
\hline \multirow[b]{2}{*}{ Laboratory vs. } & \multicolumn{4}{|c|}{ FreeStyle Precision device ${ }^{1}$} & \multicolumn{4}{|c|}{ GlucoMen LX Plus device ${ }^{2}$} \\
\hline & $\begin{array}{l}\text { Spearman } \\
\text { correlation }\end{array}$ & Med. diff. ${ }^{3}$ & $\mathrm{IQ}^{4}$ & $P$-value & $\begin{array}{l}\text { Spearman } \\
\text { correlation }\end{array}$ & Med. diff. & IQ & $P$-value \\
\hline EDTA blood & 0.94 & 0.05 & 0.14 & $<0.01$ & 0.83 & -0.30 & 0.19 & $<0.01$ \\
\hline Serum & 0.97 & 0.35 & 0.32 & $<0.01$ & 0.95 & 0.03 & 0.10 & $<0.01$ \\
\hline Plasma & 0.95 & 0.42 & 0.32 & $<0.01$ & 0.92 & 0.00 & 0.12 & 0.06 \\
\hline
\end{tabular}

${ }^{1}$ Abbott GmbH \& Co. KG, Wiesbaden, Germany.

${ }^{2}$ A. Menarini GmbH, Vienna, Austria.

${ }^{3}$ Med. diff. = median difference $(\mathrm{mmol} / \mathrm{L})$

${ }^{4} \mathrm{IQ}=$ interquartile range $(\mathrm{mmol} / \mathrm{L})$.

and 10 European countries, with the prevalence ranging from 11.2 to $55.7 \%$ (Voyvoda and Erdogan, 2010; McArt et al., 2012; Suthar et al., 2013). The lowered proportions presented in the current study might be caused by the extended time frame of blood sampling, because the prevalence of ketosis usually peaks within the first weeks of lactation (Cook et al., 2006a; Walsh et al., 2007b; Dubuc et al., 2010). Therefore, further studies are planned, focusing on fresh cow health within the first 3 wk of lactation to increase the absolute and relative number of SCK within the data set.

Comparing the test characteristics of the hand-held devices used in this study with other already evaluated devices, the FSP device showed similar test characteristics in terms of mean deviation, standard deviation, and Bland-Altman plots, whereas the GLX device showed stronger deviations. The correlation coefficient $(\mathrm{r}=$ $94 \%)$, mean absolute difference $(0.04 \mathrm{mmol} / \mathrm{L})$, and standard deviation $( \pm 0.15 \mathrm{mmol} / \mathrm{L})$ between the gold standard and the FSP device were comparable with those in the studies of Voyvoda and Erdogan (2010) using the Optium Xceed device (Abbott Diabetes Care; $\mathrm{r}=97 \%$; mean difference $=36.7 \mu \mathrm{mol} / \mathrm{L})$ and Iwersen et al. (2009) evaluating the Precision Xtra meter (r $=95 \%$ and mean difference $=-32.4 \pm 152.7 \mu \mathrm{mol} / \mathrm{L}$ in experiment 1 and $\mathrm{r}=94 \%$ and mean difference $=$ $-48.2 \pm 261.7 \mu \mathrm{mol} / \mathrm{L}$ in experiment 2 ). Whereas in studies of Iwersen et al. (2009), the Precision Xtra underestimated the laboratory results, in the current study, the FSP device slightly overestimated the BHBA concentration determined at the laboratory. These contradictory findings might be the result of the omitted calibration procedure. Using the GLX device, a lower correlation coefficient of approximately $80 \%$, with a mean difference between the device and laboratory results of $-0.12 \pm 0.22 \mathrm{mmol} / \mathrm{L}$ were determined.

From a clinical point of view, mean differences between the gold standard and the FSP device were only marginal. While interpreting the results of the GLX device, the mean difference between the device and the laboratory results should be considered.

In experiment 2, we found the highest agreement between the laboratory BHBA serum concentration and the GLX device when plasma was tested. Median differences were greatest for measurements in whole blood, causing a doubling in variance to $26.6 \%$ compared with $13.3 \%$ for the FSP device. Referring to the manufacturers' manuals, the test strips for both devices were calibrated against plasma (in human medicine). As both devices are designed as point-ofcare tests using whole blood, it is unclear if different adjustment procedures might be responsible for the differing compliance between the devices and the laboratory results.

Table 5. Results of the analysis of covariances on the differences between the BHBA concentration determined at the laboratory and results determined with FreeStyle Precision (Abbott GmbH \& Co. KG, Wiesbaden, Germany) or GlucoMen LX Plus (A. Menarini GmbH, Vienna, Austria) devices

\begin{tabular}{llrrrrr}
\hline Device & Parameter $^{1}$ & $\mathrm{~B}^{2}$ & SE & Student's $t$ & $P$-value & $95 \%$ CI \\
\hline FreeStyle Precision & Intercept & 0.420 & 0.060 & 6.530 & $<0.01$ & 0.290 to 0.540 \\
& Temp. device/strip & 0.001 & 0.003 & 0.004 & 0.99 & -0.006 to 0.006 \\
& Temp. sample & -0.012 & 0.003 & -3.922 & $<0.01$ & -0.017 to -0.006 \\
GlucoMen LX Plus & Intercept & 0.069 & 0.044 & 1.585 & 0.12 & -0.018 to 0.156 \\
& Temp. device/strip & -0.001 & 0.002 & -0.606 & 0.55 & -0.005 to 0.003 \\
& Temp. sample & -0.008 & 0.002 & -4.216 & $<0.01$ & -0.013 to -0.004 \\
\hline
\end{tabular}

${ }^{1}$ Temp. = temperature (measurements within a temperature range from $+4{ }^{\circ} \mathrm{C}$ to $32^{\circ} \mathrm{C}$ in increments of $4{ }^{\circ} \mathrm{C}$ ). The test strips used were FreeStyle Precision $\beta$-Ketone (Abbott Diabetes Care, Witney, UK) and GlucoMen LX $\beta$-Ketone Sensor (A. Menarini GmbH).

${ }^{2} \mathrm{~B}=$ changes in the difference between hand-held device and laboratory result by increasing the parameter by 1 unit. 


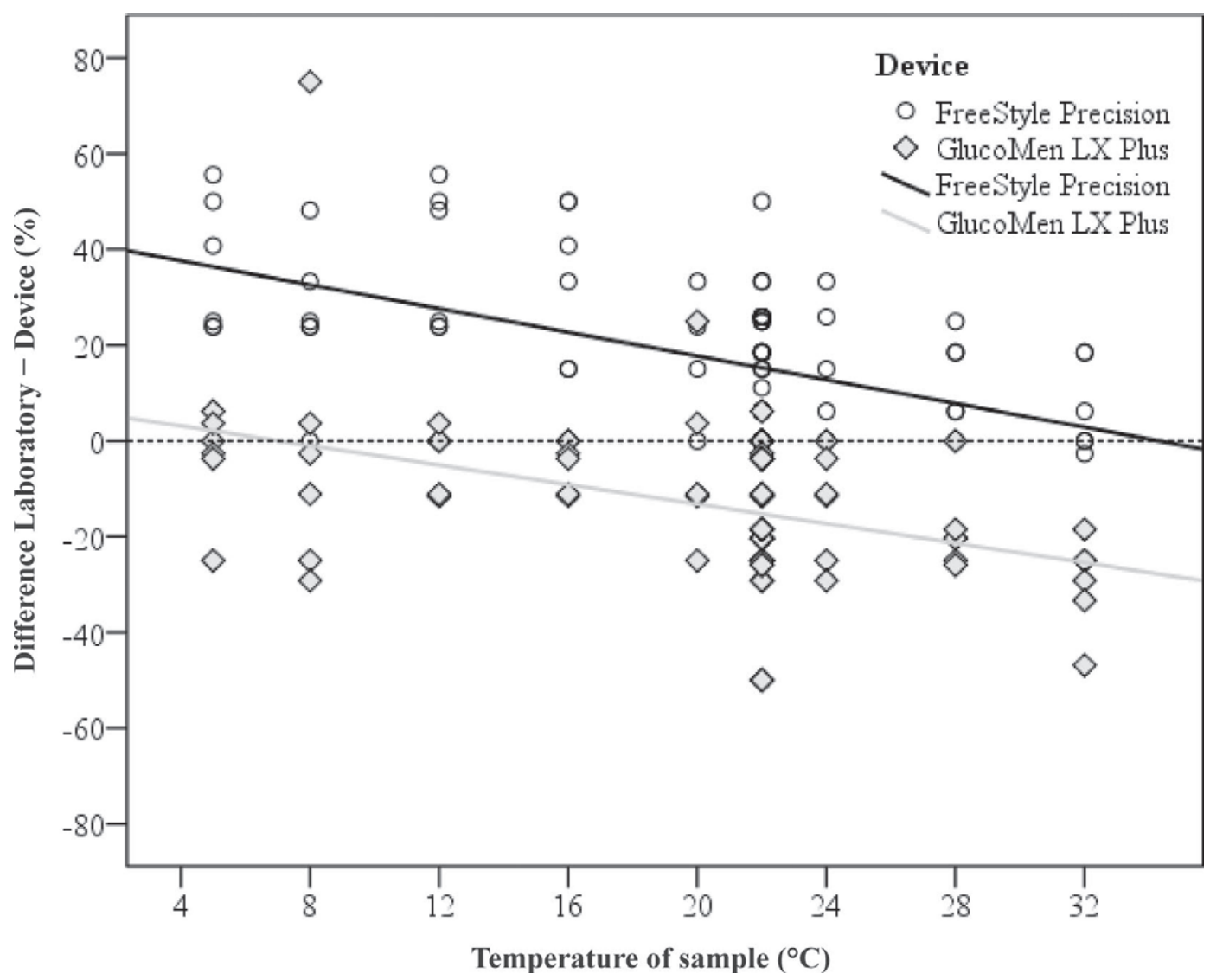

Figure 4. Influence of the sample temperature on the difference between the BHBA concentrations determined in the laboratory and with FreeStyle Precision (Abbott GmbH \& Co. KG, Wiesbaden, Germany) or GlucoMen LX Plus (A. Menarini GmbH, Vienna, Austria) devices, respectively.

For both devices, no significant differences could be detected between the BHBA concentration analyzed in whole and EDTA-added blood. Even if a tendency $(P$ $=0.06)$ exists for the FSP to underestimate the BHBA concentration analyzed in whole blood compared with EDTA-added blood, from a clinical standpoint, the mean difference of $0.02 \pm 0.08 \mathrm{mmol} / \mathrm{L}$ is irrelevant.

By performing ROC analyses, optimized decision thresholds, corresponding to serum BHBA concentrations of 1.2 and $1.4 \mathrm{mmol} / \mathrm{L}$, were determined for both devices. Compared with the gold standard, thresholds for the GLX device should be lowered by $0.1 \mathrm{mmol} / \mathrm{L}$. Referring to the established thresholds of Duffield et al. (2009), blood samples analyzed with the GLX device should, therefore, not exceed BHBA concentrations of $1.1 \mathrm{mmol} / \mathrm{L}$ in the first, and $1.3 \mathrm{mmol} / \mathrm{L}$ in the second week of lactation to prevent an increased health risk and reduced milk production.

Applying the adjusted thresholds as described above, observed Se and Sp for both hand-held devices were within the range of those in studies conducted by Voyvoda and Erdogan (2010; $1.2 \mathrm{mmol} / \mathrm{L}: \mathrm{Se}=85 \%$ and $\mathrm{Sp}=94 \% ; 1.4 \mathrm{mmol} / \mathrm{L}: \mathrm{Se}=90 \%$ and $\mathrm{Sp}=98 \%)$ and by Iwersen et al. (2009; $1.2 \mathrm{mmol} / \mathrm{L}: \mathrm{Se}=88 \%$ and $\mathrm{Sp}=96 \% ; 1.4 \mathrm{mmol} / \mathrm{L}: \mathrm{Se}=96 \%$ and $\mathrm{Sp}=97 \%$ ).

The AUC were similar for both devices using a serum concentration of $1.4 \mathrm{mmol} / \mathrm{L}$ as decision criterions for SCK, but were significantly different at a serum concentration of $1.2 \mathrm{mmol} / \mathrm{L}$ by 5 percentage points. The latter indicated a greater discriminatory power of the FSP in detection of SCK with lower BHBA concentrations in blood.

Because the chemical reactions within the test strips are based on enzymatic reactions, which in general are temperature dependent, different scenarios were simulated in this study. Both hand-held devices were initially developed for use in humans. Consequently, the devices and test strips are usually stored at room temperature. Furthermore, for diabetes monitoring in human medicine, a single blood drop is commonly obtained from the finger pad and analyzed immediately after collection, resulting in small variance of the temperature of the tested blood drop. However, because of the partial rough environment in large animal practice, 
these controlled conditions could not always have been guaranteed. Therefore, 4 scenarios in terms of storage conditions of the hand-held devices and test strips as for the blood samples were simulated. In the first scenario, blood samples were stored at different temperatures and analyzed with devices and test strips, which were stored at room temperature, representing a lagged analysis of a blood sample with a device, which, for instance, was stored in a vehicle or veterinary practice. In the second scenario, the samples, devices, and test strips were kept at various temperatures, representing a short lag in analysis (e.g., in case of group testing of animals) by keeping the samples and devices at ambient temperatures. Within the third scenario, samples, devices, and test strips were kept at room temperature, representing an immediate testing of samples (for instance, under moderate climate conditions in spring or early summer). In the fourth scenario, samples were kept at room temperature, whereas the device and test strips were stored at various temperature conditions, representing an immediate testing of samples, with the device being stored at ambient temperature (e.g., during cold winters or hot summers).

The differences between the laboratory results and the results obtained with the hand-held devices were not affected by the storage conditions of the devices and test strips, but significantly influenced by the temperature of the tested blood sample. The temperature of the blood samples within a range of +5 to $+32^{\circ} \mathrm{C}$ resulted in differences between the laboratory results and hand-held devices of approximately 30 percentage points.

For each individual hand-held meter, no differences could be observed between the BHBA concentrations determined in whole blood and EDTA blood, and in serum and plasma. The BHBA concentrations in serum and plasma, however, were significantly greater than in the corresponding whole-blood and EDTA-anticoagulated samples. These findings are contrary to the results of Stokol and Nydam (2005), reporting no differences in BHBA concentrations in whole blood compared with separated plasma or serum. In that study, samples were analyzed within $24 \mathrm{~h}$ after collection at 4 or $24^{\circ} \mathrm{C}$ by an automated wet chemistry analyzer. In our study, whole blood and EDTA-anticoagulated blood were tested with both hand-held meters immediately after sampling at a sample temperature of approximately $32^{\circ} \mathrm{C}$. Serum and plasma BHBA concentrations were tested with both devices about $2 \mathrm{~h}$ after sampling at room temperature $\left(\sim 22^{\circ} \mathrm{C}\right)$. Referring to our results of experiment 3 , a difference of $10^{\circ} \mathrm{C}\left(+32\right.$ to $\left.+22^{\circ} \mathrm{C}\right)$ in the temperature of the tested samples led to a difference between the laboratory and the results of the hand-held devices of approximately $20 \%$. Therefore, the effect of the sample temperature causing the differences in BHBA concentrations between whole blood and serum or EDTA blood and plasma is the most likely explanation.

\section{CONCLUSIONS}

The FSP meter showed excellent test characteristics for a hand-held device, comparable with the previously evaluated device Precision Xtra. The hand-held meter GlucoMen LX Plus showed lower agreement with the laboratory results, but after adjusting the thresholds for SCK, as determined by ROC analyses, Se and Sp were considered as good. Testing serum or plasma with both electronic devices resulted in considerably greater BHBA concentrations compared with whole blood or EDTA-anticoagulated blood. Storage of devices and test strips within a temperature range of +5 to $+32^{\circ} \mathrm{C}$ did not influence the determined test results. In contrast, the difference between the results of the devices and the laboratory results were significantly influenced by the temperature of the tested sample. Therefore, when using the hand-held devices for cowside testing, the type and temperature of the tested blood sample should be considered. Furthermore, we encourage the manufacturers to improve the suitability of the devices (for instance, by implementing temperature-dependent adaption procedures of the displayed results).

\section{ACKNOWLEDGMENTS}

We acknowledge the financial support by A. Menarini GmbH (Vienna, Austria). Samples were processed and results evaluated uninfluenced and independently by the sponsor. The authors gratefully acknowledge the cooperation with the staff of the cooperating study sites and the Central Diagnostic Unit of the University of Veterinary Medicine, Vienna (Austria).

\section{REFERENCES}

Bauman, D. E., and W. B. Currie. 1980. Partitioning of nutrients during pregnancy and lactation: A review of mechanisms involving homeostasis and homeorhesis. J. Dairy Sci. 63:1514-1529.

Bell, A. W. 1995. Regulation of organic nutrient metabolism during transition from late pregnancy to early lactation. J. Anim. Sci. 73:2804-2819.

Bland, J. M., and D. G. Altman. 1986. Statistical methods for assessing agreement between two methods of clinical measurement. Lancet 1:307-310.

Carrier, J., S. Stewart, S. Godden, J. Fetrow, and P. Rapnicki. 2004. Evaluation and use of three cowside tests for detection of subclinical ketosis in early postpartum cows. J. Dairy Sci. 87:3725-3735.

Chapinal, N., S. J. LeBlanc, M. E. Carson, K. E. Leslie, S. Godden, M. Capel, J. E. P. Santos, M. W. Overton, and T. F. Duffield. 2012. Herd-level association of serum metabolites in the transition period with disease, milk production, and early lactation reproductive performance. J. Dairy Sci. 95:5676-5682.

Cook, N., G. Oetzel, and K. Nordlund. 2006a. Modern techniques for monitoring high-producing dairy cows 1. Principles of herd-level diagnoses. In Pract. 28:510-515. 
Cook, N., G. Oetzel, and K. Nordlund. 2006b. Modern techniques for monitoring high-producing dairy cows 2. Practical applications. In Pract. 28:598-603.

Dubuc, J., T. F. Duffield, K. E. Leslie, J. S. Walton, and S. J. LeBlanc. 2010. Risk factors for postpartum uterine diseases in dairy cows. J. Dairy Sci. 93:5764-5771.

Duffield, T. F., K. D. Lissemore, B. W. McBride, and K. E. Leslie. 2009. Impact of hyperketonemia in early lactation dairy cows on health and production. J. Dairy Sci. 92:571-580.

Duffield, T. F., D. Sandals, K. E. Leslie, K. Lissemore, B. W. McBride, J. H. Lumsden, P. Dick, and R. Bagg. 1998. Efficacy of monensin for the prevention of subclinical ketosis in lactating dairy cows. J. Dairy Sci. 81:2866-2873.

Geishauser, T., K. Leslie, D. Kelton, and T. Duffield. 1998. Evaluation of five cowside tests for use with milk to detect subclinical ketosis in dairy cows. J. Dairy Sci. 81:438-443.

Geishauser, T., K. Leslie, J. Tenhag, and A. Bashiri. 2000. Evaluation of eight cow-side ketone tests in milk for detection of subclinical ketosis in dairy cows. J. Dairy Sci. 83:296-299.

GfE (Gesellschaft für Ernährungsphysiologie). 2001. Empfehlungen zur Energie- und Nährstoffversorgung der Milchkühe und Aufzuchtrinder. DLG-Verlag, Frankfurt (Main), Germany.

Grummer, R. R. 2008. Nutritional and management strategies for the prevention of fatty liver in dairy cattle. Vet. J. 176:10-20.

Grummer, R. R., D. G. Mashek, and A. Hayirli. 2004. Dry matter intake and energy balance in the transition period. Vet. Clin. North Am. Food Anim. Pract. 20:447-470.

Hayirli, A., R. R. Grummer, E. V. Nordheim, and P. M. Crump. 2002. Animal and dietary factors affecting feed intake during the prefresh transition period in Holsteins. J. Dairy Sci. 85:3430-3443.

Herdt, T. H. 2000. Ruminant adaptation to negative energy balance. Influences on the etiology of ketosis and fatty liver. Vet. Clin. North Am. Food Anim. Pract. 16:215-230.

Heuwieser, W., U. Falkenberg, M. Iwersen, R. Voigtsberger, and W. Padberg. 2007. Evaluation and use of an automated human betahydroxybutyrate (BHBA) test for cowside detection of subclinical ketosis in dairy cattle. Pages 253-254 in Proc. 40th Annu. Conv. Am. Assoc. Bov. Pract., Vancouver, BC. Am. Assoc. Bov. Pract., Stillwater, OK.

Iwersen, M., U. Falkenberg, R. Voigtsberger, D. Forderung, and W. Heuwieser. 2009. Evaluation of an electronic cowside test to detect subclinical ketosis in dairy cows. J. Dairy Sci. 92:2618-2624.

Jeppesen, R., J. M. Enemark, and C. Enevoldsen. 2006. Ketone body measurement in dairy cows. Pages OS 43-42 in Proc. 24th World Buiatrics Congress, Nice, France. World Assoc. Buiatrics, Vienna, Austria.

LeBlanc, S. 2010. Monitoring metabolic health of dairy cattle in the transition period. J. Reprod. Dev. 56:S29-S35.
McArt, J. A. A., D. V. Nydam, and G. R. Oetzel. 2012. A field trial on the effect of propylene glycol on displaced abomasum, removal from herd, and reproduction in fresh cows diagnosed with subclinical ketosis. J. Dairy Sci. 95:2505-2512.

Oetzel, G. R. 2004. Monitoring and testing dairy herds for metabolic disease. Vet. Clin. North Am. Food Anim. Pract. 20:651-674.

Ospina, P. A., D. V. Nydam, T. Stokol, and T. R. Overton. 2010 Evaluation of nonesterified fatty acids and $\beta$-hydroxybutyrate in transition dairy cattle in the northeastern United States: Critical thresholds for prediction of clinical diseases. J. Dairy Sci. 93:546-554.

Panousis, N., C. Brozos, I. Karagiannis, N. D. Giadinis, S. Lafi, and M. Kritsepi-Konstantinou. 2012. Evaluation of Precision Xceed ${ }^{\circledR}$ meter for on-site monitoring of blood $\beta$-hydroxybutyric acid and glucose concentrations in dairy sheep. Res. Vet. Sci. 93:435-439.

Pichler, M., J. Gasteiner, A. Damberger, M. Iwersen, and M. Drillich. 2012. Evaluation of an electronic hand-held meter for measurement of beta-hydroxybutyrate (BHB) in ewes. Reprod. Domest. Anim. 47(Suppl. 5):69.

Quiroz-Rocha, G. F., S. J. LeBlanc, T. F. Duffield, D. Wood, K. E. Leslie, and R. M. Jacobs. 2009. Reference limits for biochemical and hematological analytes of dairy cows one week before and one week after parturition. Can. Vet. J. 50:383-388.

Stokol, T., and D. V. Nydam. 2005. Effect of anticoagulant and storage conditions on bovine nonesterified fatty acid and beta-hydroxybutyrate concentrations in blood. J. Dairy Sci. 88:3139-3144.

Suriyasathaporn, W., A. J. J. M. Daemen, E. N. NoordhuizenStassen, S. J. Dieleman, M. Nielen, and Y. H. Schukken. 1999. $\beta$-Hydroxybutyrate levels in peripheral blood and ketone bodies supplemented in culture media affect the in vitro chemotaxis of bovine leukocytes. Vet. Immunol. Immunopathol. 68:177-186.

Suthar, V. S., J. Canelas-Raposo, A. Deniz, and W. Heuwieser. 2013 Prevalence of subclinical ketosis and relationships with postpartum diseases in European dairy cows. J. Dairy Sci. 96:2925-2938.

Swets, J. A. 1988. Measuring the accuracy of diagnostic systems. Science 240:1285-1293.

Voyvoda, H., and H. Erdogan. 2010. Use of a hand-held meter for detecting subclinical ketosis in dairy cows. Res. Vet. Sci. 89:344351.

Walsh, R. B., D. F. Kelton, T. F. Duffield, K. E. Leslie, J. S. Walton, and S. J. LeBlanc. 2007a. Prevalence and risk factors for postpartum anovulatory condition in dairy cows. J. Dairy Sci 90:315-324.

Walsh, R. B., J. S. Walton, D. F. Kelton, S. J. LeBlanc, K. E. Leslie, and T. F. Duffield. 2007b. The effect of subclinical ketosis in early lactation on reproductive performance of postpartum dairy cows. J. Dairy Sci. 90:2788-2796. 